\title{
APPLICATION OF LINEAR PROGRAMMING FOR DEVELOPING DISTRIBUTION OPTIMIZATION MODEL FOR SERVICES: A CASE STUDY OF HOSPITAL SPECIALITY SERVICES DISTRIBUTION
}

\author{
Devi Prasad Ghosh ${ }^{1 *}$ \\ ${ }^{*}$ Head of Marketing Department, Institute of Management Technology (IMT), Hyderabad. \\ Director, Graph Matrix Consulting, Hyderabad \\ Email:devghosh@imthyderabad,edu.in/dp_ghosh@graphmatrix.com/dp_ghosh@yahoo.com \\ Mobile: +919324705051
}

*Corresponding Author: -

Email:devghosh@imthyderabad,edu.in

\begin{abstract}
: -
The objective of the paper was to extend the tangible goods distribution model to services and develop a decisionmaking tool that services distribution company such as hospital chain could use to derive a more efficient and effective distribution of scarce and expensive resources of specialists to its hospitals. The paper attempts to optimize the creation of speciality services infrastructure at a hospital location and planning for specialists' resources. The paper developed the problem definition, the formulation of Linear Programming Model, the procedure is designed as a solution to the problem, and the outcome as benefits to the company is presented. Linear programming model is formulated that can be solved using commercial software. This paper demonstrates the potential for using linear programming in managing large-scale distribution problems for services. The model resulted in the creation of new metrics for measuring the performance for services distribution, the model will help the company understand conditions that results in hospital specialty services shortages, and the model lead to the discovery of inefficiencies in company speciality services distribution strategy. The model is directly beneficial to any company in hospital services distribution particularly speciality or super-speciality services. With a large proportion of speciality services sales is accounted by referral of physicians direct to customers, the distribution of speciality procedure services not only defines the cost but also customer satisfaction by managing services delivery quality \& time and return- of-patients for post procedure checkups. The model is also applicable for cases in any other services industries which operates in multiple markets and have large variety of Services Units.
\end{abstract}

Keywords: Services Distribution Optimization Model. Strategic Planning. Linear Programming Model, Hospital Speciality Services Distribution, Service Strategy.

\section{(a) $\$(1)$}




\section{INTRODUCTION}

The purpose of formulating and optimizing the services distribution model for medical specialists' distribution do provide company with a means for comparing their strategy for moving medical specialists through their hospital distribution network with the optimized strategy derived from the formulated model. Such analyses usually lead to a more economical services distribution strategy. Therefore, the first objective of the paper was to establish the scope of the model by identifying the aspects of the company services distribution that would be studied. The second objective was to define the decision variables, parameters, constraints and performance measures necessary for formulating a model of company medical specialists' distribution operations. The third objective was to formulate a linear programming model for the sales \& services distribution strategy and planning. And the fourth objective was to help analyze the optimized distribution of medical specialists determined by the model and identify improvements to the existing speciality procedure services sales \& distribution strategy used by the company.

Being a business partner associated with speciality medical procedure business of the company helped in establishing the problem definition (project scope); defining the model's decision variables, parameters, constraints, and performance measures; validating the model formulation; providing input parameters for the model; and defining and analyzing test scenarios. The close involvement with the process helped in quantifying the variables and relationships, which drive medical specialists' distribution and identifying areas for additional data collection and study.

\section{The Company Background}

Apollo Hospitals is Asia's largest and most trusted healthcare group and its presence includes 9,215 beds across 64 Hospitals, 2,200 Pharmacies, over 90 Primary Care and Diagnostic Clinics, 110 plus Telemedicine Centers and 80 plus Apollo Munich Insurance branches panning the length and breadth of the Country. As an integrated healthcare service provider with Health Insurance services, Global Projects Consultancy capability, 12 plus medical education centers and a Research Foundation with a focus on global Clinical Trials, epidemiological studies, stem cell \& genetic research, Apollo Hospitals has been at the forefront of new medical breakthroughs with the most recent investment being that of commissioning the first Proton Therapy Center across Asia, Africa and Australia in Chennai, India. (Source: company website www.apollohospitals.com)

\section{Problem Definition}

Like most Health Care services the company is following currently Hub and Spoke distribution model for specialists. The business is divided into four regions India East \& Bangladesh India West, India North, India South \& Gulf. Calcutta, Mumbai, Delhi \& Chennai city act as gateway of specialists for respective region. Hospitals at Kolkata, Mumbai, Delhi and Chennai acts as Regional Hub Hospitals. Regional hospitals falling under a region are serviced for all products \& services through this Regional Hub hospitals. This service distribution model is designed based on operational convenience of the company and not on scientific method of market demand or cost efficiency of service distribution. In the first instant, we can conclude that the Hub and Spoke model in the current form described here may fulfill administrative requirements based on geography control, but may not be the optimum model for distribution of hospital services particularly high cost speciality and super-speciality medical procedures. Moreover, this does not take into account the advantages of transshipments model where inter-hospital transfers of specialists or high value services with limited demand can be designed in hospitals as per market need.

The company currently markets many speciality and super-speciality services. Because each speciality and superspeciality medical procedures have different Medical Procedure Durations, different Prices, has different Demand and Operating Cost, they are treated as separate service units (SUs) in the model. The data for 18 varieties of key Speciality Medical Procedures provided here is used as a representation for the understanding of the model. (Table 1)

Company has hospitals throughout the country, Bangladesh, Gulf and all hospitals are used to build specific speciality infrastructure and distribute the company speciality product. For the purpose of this project, it was decided that 18 locations of hospital units currently in operation based on state/ region/ country would be included in the model. Also, only speciality and super- speciality procedures and not general surgery procedures will be considered for the study as it approximately constitute more than two-third of the operations facility cost of the hospital. The locations of the hospital units are provided. (Table 2)

The transfer of specialists and hospital services distribution is a dynamic activity. The transfer and distribution activities are indexed over time in the model. The model must take into consideration the planning horizon of the company. The term time bucket was used to describe a 1-month period. The sales period for the company is 12 months as per Indian Financial Year (April to March), that is 12 time buckets. Demand and transfer numbers are based on hospital unit sales team estimates for the year that decrease in validity over extended planning horizons. The model takes into account the transfer planning horizon of 3 months followed by the company. The shorter planning horizon increased the accuracy of the forecasted input data. Therefore, the estimates in the model need to be updated after every quarter for subsequent quarter to improve the accuracy of the model.

The Medical Procedure Service was used by the company as a service unit to count the movements of specialists. Company generally collects and records data on No.of specialist per medical procedure basis, but services is almost always distributed in full as Medical Procedure and Specialists services. The model indicate appropriate decision's regarding moving medical specialists between hospital units. The model recorded the ending service inventory, sales and lost sales for every time period. These quantities are available in No. of service units (Medical Procedure Service) In order to facilitate model development, Company need to estimate several physical and financial parameters. The physical parameters include beginning service inventory levels, transfer levels and demand levels for each Service 
Units (indexed by time bucket where appropriate), as well as medical procedure capacities for each hospital unit. Company also need to estimate the financial parameters include selling price as well as transfer, handling and hosting costs for a medical procedure. All financial parameters were measured on a per service unit basis (or per service unit per time bucket).

Based on general company practice, four key assumptions were made for medical specialist transfer and services distribution. First, demand and transfer forecasts made were accurate. Second, specialists transfer cost could be captured on a per medical procedure basis. Third, adequate infrastructure facilities is always available for transfer of specialists. Fourth, fractional transfer values such as less than one complete procedure are not captured in the model. All of these assumptions are necessary to simplify the model. A simpler model would be beneficial for this study for easier understanding of the reader and practitioners for implementation.

\section{Linear Programming Model Formulation}

The first step in formulating the linear programming (LP) model is to establish the indexes over which the variables and parameters of the model will be defined. The indexes are Speciality/ Super-speciality Procedure Service Unit, hospital units and time bucket.

$\begin{array}{ll}\text { Specialty Procedures (Service Unit): } & S=1,2, \ldots \ldots, 18 \ldots \ldots \text {. }(\text { Table } 1) \\ \text { Hospital Unit: } & H=1,2, \ldots, 18, \ldots \ldots \text { (Table 2) } \\ \text { Time bucket: } & T=1,2,3, \ldots .12 \ldots \ldots \ldots \text {. }\end{array}$

Having defined these indexes, the next step is to identify the variables included in the model. These variables include the decision variables and other output variables.

The decision variables in this model captures the movement of medical specialists among company hospitals. Therefore, the decision variables are:

$n P_{\text {sijt }}$ The number of Specialists for Medical Procedure Service $s$ delivered from hospital unit i to hospital unit $j$ in time bucket $t$.

The output variables are period end service procedure inventory, new sales, lost sales at the hospital. Therefore, the output variables are:

$n I_{\text {sit }} \quad$ The number of medical procedure services $s$ remaining in service inventory at hospital unit i at the end of time bucket $t$

$n S_{\text {sit }} \quad$ The number of medical procedure services $s$ sales at hospital units $i$ during time bucket $t$

$n L S_{\text {sit }} \quad$ The number of medical procedure services $s$ that were not available for sale upon demand at hospital unit i during time bucket $t$

The input parameters for the model include the physical and financial parameters. The physical parameters are:

$n I_{s i, t-1} \quad$ The number of medical procedure services $s$ in Service Inventory at hospital unit $I$ prior to the time bucket $\mathrm{t}$.

$n S C_{i} \quad$ The number of medical procedure services capacity of hospital unit $i . n S h_{\text {sit }}$ The number of medical procedure services $s$ at hospital unit $i$ that become available for sale during time bucket $t$

$n D_{\text {sit }} \quad$ The number of service unit of Demand for medical procedure services $s$ at hospital unit $i$ during time bucket $t$.

The cost parameters are:

SP $P_{s i} \quad$ The selling price of a medical procedure services $s$ at hospital unit i

Ctij The cost to transfer one medical specialist from hospital unit $i$ to hospital unit $j$

Chi The cost to handle one medical procedure service at hospital unit $i$

Csi The cost to support one medical specialist at hospital unit $i$ for one time bucket The model objective is to identify the most efficient decisions regarding the distribution of medical specialist. Therefore, the objective function was defined to maximize the profit margin that is the difference of sales revenue generated and the costs to transfer specialist, handle procedure and support of specialist.

Maximize

$$
\sum_{s=1}^{18} \sum_{i=1}^{18} \sum_{t=1}^{12} S P_{s i} n S_{s, i, t}-\sum_{s=1}^{18} \sum_{i=1}^{18} \sum_{j=1}^{18} \sum_{t=1}^{12}\left(C t_{i, j}+C h_{i}\right) n P_{s, i, i, t}-\sum_{s=1}^{18} \sum_{i=1}^{18} \sum_{t=1}^{12} C s_{s, i, t} n I_{s, i, t}
$$


There are several functional relationships, which limit the values that can be taken on by the decision and output variables. The first of these relationships requires that balance be maintained between medical procedure services input to a hospital unit during a time bucket (initial service inventory, transfer in from hospital unit, new hire or outsourcing from outside), medical specialists sent out of a hospital unit during a time bucket (transfer out, sales), and ending services inventory.

$$
n I_{s, t-1}+\sum_{i=1}^{18} n P_{s, i, j, t}+n P_{s, i, t}-\sum_{i=1}^{18} n S h_{s, i, j, t}-n S_{s, i, t}=n I_{s, i, t} \quad \forall s, i, t
$$

The ending service inventory for time bucket $\mathrm{t}-1$ corresponds to beginning service inventory for time bucket $\mathrm{t}$.

$$
n I_{s, i, t-1}=b_{-} I_{s, i, t} \quad \forall s, i, t
$$

The second functional relationship states that the total service inventory at a hospital unit be at or below the capacity of that hospital unit.

$$
\sum_{s=1}^{18} n I_{s, i, t} \leq n S C_{i} \quad \forall i, t
$$

The third functional relationship states that all demand is accounted for by either a sale or a lost sale.

$$
n S_{s, i, t}+n L S_{s, i, t}=n D_{s, i, t} \quad \forall s, i, t .
$$

Adding non-negativity constraints for each of the decision and output variables yields the final formulation.

Maximize

s.t.

$$
n I_{s, i, t-1}+\sum_{i=1}^{18} n P_{s, i, j, t}+n P_{s, i, t}-\sum_{i=1}^{18} n P_{s, i, j, t}-n S_{s, i, t}=n I_{s, i, t} \quad \forall s, i, t
$$

$$
\begin{array}{cll}
n I_{s, i, t-1}+\sum_{j=1}^{18} n P_{s, i, j, t}+n P_{s, i, t}-\sum_{i=1}^{18} n P_{s, i, j, t}-n S_{s, i, t}= & n I_{s, i, t} \\
\sum_{s=1}^{18} n I_{s, i, t} \leq n S C_{i} & \forall s, i, t \\
n S_{s, i, t}+n L S_{s, i, t}=n D_{s, i, t} & \forall s, i, t \\
n P_{s, i, j, t} \geq 0 & \forall s, i, j, t \\
n I_{s, i, t} \geq 0 & \forall s, i, t \\
n L S_{s, i, t} \geq 0 & \\
n S_{s, i, t} \geq 0 & \forall s, i, t
\end{array}
$$

Above formulation is a linear programming problem having multiple variables and multiple functional constraints. The matrix below represents the specialist transfer cost between hospital unit $\mathrm{i}$ and hospital unit $\mathrm{j}$ for number of medical procedure services $\mathrm{nPi}$

\begin{tabular}{|l|l|l|l|l|l|}
\hline $\begin{array}{l}\text { Hospital Units } \\
\text { (i j) }\end{array}$ & $\mathrm{H}_{1}$ & $\mathrm{H}_{2}$ & $\mathrm{H}_{3}$ & $\mathrm{H}_{\mathrm{j}}$ & $\begin{array}{l}\text { Total Hospital } \\
\text { Units }\end{array}$ \\
\hline $\mathrm{H}_{1}$ & $\mathrm{Ct}_{11}$ & $\mathrm{Ct}_{12}$ & $\mathrm{Ct}_{13}$ & $\mathrm{Ct}_{1 j}$ & $\mathrm{nP}_{1}$ \\
\hline $\mathrm{H}_{2}$ & $\mathrm{Ct}_{21}$ & $\mathrm{Ct}_{22}$ & $\mathrm{Ct}_{23}$ & $\mathrm{Ct}_{2 j}$ & $\mathrm{nP}_{2}$ \\
\hline $\mathrm{H}_{3}$ & $\mathrm{Ct}_{31}$ & $\mathrm{Ct}_{32}$ & $\mathrm{Ctt}_{33}$ & $\mathrm{Ct}_{3 j}$ & $\mathrm{nP}_{3}$ \\
\hline $\mathrm{H}_{\mathrm{i}}$ & $\mathrm{Ct}_{11}$ & $\mathrm{Ct}_{22}$ & $\mathrm{Ct}_{\mathrm{i}}$ & $\mathrm{Ct}_{\mathrm{j}}$ & $\mathrm{nP}_{\mathrm{i}}$ \\
\hline Total Hospital Units & $\mathrm{nP}_{1}$ & $\mathrm{nP}_{2}$ & $\mathrm{nP}_{3}$ & $\mathrm{nP}_{\mathrm{j}}$ & $\sum \mathbf{n P}_{\mathbf{j}}$ \\
\hline
\end{tabular}




\section{Solution And Implementation}

Having formulated the distribution problem as a linear programming problem, the next step in the project is to select a method of performing the optimization. LINGO (developed by LINDO Systems, Inc.) is a suitable for use and readily available. It permits the use of spreadsheets for reading the values of input parameters and output variables. The input and output spreadsheets data for the model are provided by the company. For the purposes of debugging and testing the LINGO code, a test problem can be defined. Once the LINGO code is verified, company input data can be used for implementation.

\section{Managerial Use}

The output of the model can be compared to the actual decisions to identify weaknesses in any distribution strategy. The process of formulating the model, developing test scenarios, and making these comparisons can provide valuable benefit to the company. In fact, six key benefits of the model that can be identified are as below:

1. The model input required that company project availability for transfer on a medical procedure services unit (SU) basis. Company had to analyze work order completions and add the one month target release cycle to determine projected medical specialist availability for transfer schedule creation. Company currently does not capture data as a formal report or tool in place; however, company recognized a need and the potential value for such data reporting.

2 The model can calculate projected revenues from sales that were transfer to meet demand based upon the selling price on a service unit basis. The model can also calculate costs for transferring specialist, medical procedure and hosting specialist Company will be able to create a ratio of transfer specialist, medical procedure and hosting costs as a percentage of revenue. Company has not historically performed this comparison in their business; however, it is a valuable measure.

3. The model calculates "lost sales" $\square$ units of sales lost due to medical specialist unavailability in the desired time bucket or transfer costs in excess of revenue from sale. In reality, company as a practice never holds a specialist or misses a sale because the revenue may not exceed the transfer costs. However, this information will be tremendously valuable as company can use it to analyze why specialist was not available or available closer to the point of sale. The model does provide information to distinguish which situation (unavailable or prohibitive transfer cost) created the lost sale situation.

4. The model assigns demand to a specific hospital unit. All specialist transfer orders dispatched to assigned hospital unit are assumed as demand for the hospital. This concept of assigning demand for particular speciality medical procedure to specific hospital unit forces sales team to improve the accuracy of demand forecast for the company.

5. The decision to run "actuals" (real data) through the model will prove to be a beneficial exercise for company. This model will for a model of benchmark of cost optimization for medical specialist transfer between hospital units. Also, the potential to report inaccurate numbers can be corrected due to review of model output.

6. The cost information is likely to reinforced two things: (1) validation for company transfer account structure, and (2) necessity of cost-sensitive measures and goals.

7. This model is will also help reduce specialists holding in regional hub hospitals.

8. It will also help in planning shifting of less demanded medical specialists housed in various hospital units to other hospital units where demand for the service exists.

\section{Conclusions}

The process of formulating the model, testing the model, and analyzing the model's results will provide valuable insights to the company. The company can decide based on the model, focus areas on their transportation and distribution processes. The implementation of the changes based on the model will result in significant cost saving due to reduction in movement of specialists. The medical specialist's movement reduction based on the model will help improve the efficiency of the services distribution system and ability of the company to improve availability of the medical specialists in the right market at right time.

Table 1: Medical Speciality Procedures (Service Units)

\begin{tabular}{|c|c|c|c|}
\hline S.No. & Medical Speciality Procedures & S.No. & Medical Speciality Procedures \\
\hline 1 & IVF \& Infertility Treatment & 10 & Knee Replacement Surgery \\
\hline 2 & Cosmetic / Plastic Surgery & 11 & Orthopedic Surgery \\
\hline 3 & Bone Marrow Transplant & 12 & Nephrology \& Urology \\
\hline 4 & Oral \& Maxillofacial Surgery & 13 & Bariatric Surgery \\
\hline 5 & Hand Micro Surgery & 14 & Cardiology \\
\hline 6 & G Scan - Open Standing MRI Scan & 15 & Gastroenterology \\
\hline 7 & 320 Slice CT Scan & 16 & Spine Surgery \\
\hline 8 & Hip Arthroscopy & 17 & Cancer/Oncology \\
\hline 9 & Minimally Invasive Cardiac Surgery & 18 & Neurology \& Neurosurgery \\
\hline
\end{tabular}


Table 2: Company Hospital Locations:

\begin{tabular}{|l|l|l|l|l|l|}
\hline S.No. & $\begin{array}{l}\text { Hospital } \\
\text { Units }\end{array}$ & $\begin{array}{l}\text { States/ } \\
\text { Region }\end{array}$ & S.No. & Hospital Units & States/ Region \\
\hline 1 & Bengaluru & Karnataka & 10 & Indore & $\begin{array}{l}\text { Madhya } \\
\text { Pradesh }\end{array}$ \\
\hline 2 & Chennai & Tamil Nadu & 11 & Guwahati & North East \\
\hline 3 & Delhi & NCR & 12 & Madurai & Tamil Nadu \\
\hline 4 & Hyderabad & Telangana & 13 & Mysore & Karnataka \\
\hline 5 & Kolkata & West Bengal & 14 & Nashik & Maharashtra \\
\hline 6 & Mumbai & Mumbai City & 15 & Dhaka & Bangladesh \\
\hline 7 & Pune & Maharashtra & 16 & Visakhapatnam & Andhra Pradesh \\
\hline 8 & Ahmedabad & Gujarat & 17 & Bilaspur & Chhattisgarh \\
\hline 9 & Bhubaneshwar & Orissa & 18 & Muscat & Gulf \\
\hline
\end{tabular}

\section{Bibliography:}

[1].Anderson D.R., Seweeney D.J. \& Williams T.A. (2001). "Quantitative Methods of Business" $8^{\text {th }}$ edition, Thompson South-Western College publishing Bangalore (India), pp 216 - 477

[2]. "Distribution Optimization Model: A Case Study of Mobile Handset Distribution System". Journal of Advance Research in Business, Management and Accounting”. April,2017, ISSN: 2456-3544.

[3].Problem definition from services distribution system observational study and analysis of Apollo Hospitals Enterprise Ltd. as a business partner with Aircel Cellular, Head - Marketing (General Manager) from May, 2010 to April., 2012.

[4].Company information validated from its official website link accessed on $9^{\text {th }}$ Sept, 2018: https://www.apollohospitals.com. 